\title{
ERRATUM to: Dying Safely
}

Magnolia Cardona-Morrell and Ken Hillman

\section{Erratum to:}

Chapter 27 in: M.A. DeVita et al. (eds.), Textbook of Rapid Response Systems, DOI 10.1007/978-3-319-39391-9_27

Chapters 27 was originally published with an incorrect figure. The chapter has been updated with the correct figure. 
Months/weeks prior to death

Severe fatigue, severe pain, anorexia, cachexia

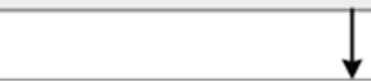

\section{Days/hours before death}

Drowsiness, irregular breathing, acrocyanosis, respirations with mandibular movements, dehydration, confusion,

delirium, loss of consciousness, and radial pulseness, death rattle

Do:

- Oral or parenteral opioids for dyspnea

- Titration of opioids for physical pain

- Antimuscarinics for excess respiratory secretions

- Benzodiazepines, antipsychotics and phenotiazines for delirium

- Midazolam and barbiturates for palliative sedation

- Discontinue dialysis

- Psychosocial support for family distress
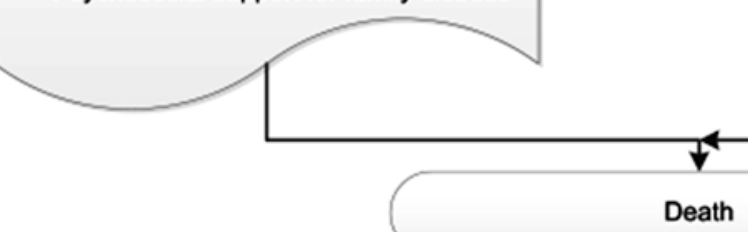

Fig. 27.1 To treat or not to treat: Journey from signs and symptoms to safe death

Death

\section{Don't:}

- Routine artificial nutrition

- Restrain agitated patient

- Oxygen therapy for hypercarbia

- Initiate chemotherapy

- Blood transfusion

- Nebulized opioids for dyspnea

- Ventilatory support/tracheostomy

- Intensive care procedures

- Attempt resuscitation

- Unsolicited information to families

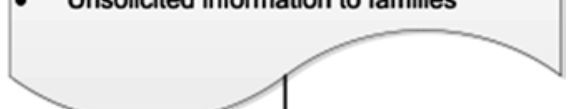

